\title{
Razumikhin-type theorem and mean square asymptotic behavior of the backward Euler method for neutral stochastic pantograph equations
}

\section{Zhanhua Yu*}

"Correspondence:

yuzhanhua@yeah.net Department of Mathematics, Harbin Institute of Technology, Weihai, 264209, P.R. China

\begin{abstract}
The Razumikhin-type stability theorems of neutral stochastic functional differential equations (NFDEs) were investigated by several authors, but there was almost no Razumikhin-type theorems on the general asymptotic stability of NFDEs with infinite delay. This paper investigates the Razumikhin-type pth moment asymptotic stability of neutral stochastic pantograph equations (NSPEs). Sufficient conditions of the pth moment asymptotic stability for NSPEs are obtained. The NSPE is a special class of NFDEs with infinite delay. We should emphasize that the Razumikhin-type theorem of this paper is established without taking difficulties from infinite delay into account. We also develop the backward Euler method for NSPEs. We prove that the backward Euler method can preserve the asymptotic behavior of the mean square stability of exact solutions under suitable conditions. Numerical examples are demonstrated for illustration.
\end{abstract}

MSC: 65C30;65L20;60H10

Keywords: neutral stochastic pantograph equations; Razumikhin-type theorem; $p$ th moment asymptotic stability; backward Euler method; mean square stability

\section{Introduction}

Neutral stochastic functional differential equations (NSFDEs) can be used to model various phenomena and processes in the field of the chemical-engineering and aero elasticity. Stability analysis of NSFDEs has attracted increasing attention; for instance, see [1-5]. Among the methods contributed to the study of stability for NSFDEs, the Razumikhin technique is a powerful and effective method. In this paper, we consider the following neutral stochastic pantograph equation (NSPE):

$$
d[x(t)-N(x(q t))]=f(t, x(t), x(q t)) d t+g(t, x(t), x(q t)) d B(t),
$$

where $0<q<1$ NSPE (1) is an important extension of stochastic pantograph equations (see [6, 7]), and NSPE (1) stems from neutral pantograph equations (NPEs). NPEs play important roles in mathematical and industrial problems (see [8]). Many authors have studied NPEs numerically and analytically. We refer the reader to [8-14]. One class of

\section{Springer}

(c) 2013 Yu; licensee Springer. This is an Open Access article distributed under the terms of the Creative Commons Attribution License (http://creativecommons.org/licenses/by/2.0), which permits unrestricted use, distribution, and reproduction in any medium, provided the original work is properly cited. 
NPEs reads

$$
[x(t)-N(x(q t))]^{\prime}=f(t, x(t), x(q t) .
$$

Taking the environmental disturbances into account, we are led to NSPE (1). The almost sure asymptotic stability of Eq. (1) has been studied (please see [15]). In this paper, we aim to study the $p$ th moment asymptotic stability of Eq. (1) by using the Razumikhin technique.

The Razumikhin technique has been successfully applied to study the stability of various NSFDEs (see, e.g., [1-3, 16-20]). Recently, Wu et al. [17] studied the $\psi^{\gamma}$ stability of the following NSFDE with unbounded delay:

$$
d\left[x(t)-N\left(x_{t}\right)\right]=f\left(t, x_{t}\right) d t+g\left(t, x_{t}\right) d B(t)
$$

by using the Razumikhin technique, where $x_{t}=\{x(t+\theta):-\infty<\theta \leq 0\}$, and some techniques are adopted to overcome the difficulties from infinite delay. Note that Eq. (1) is also a class of NSFDEs with time delay $\tau(t)=(1-q) t \uparrow \infty$ as $t \rightarrow \infty$. For given $t>0$, it is easy to see that the solution $x(t)$ of Eq. (1) depends on states of $[q t, t]$, but the solution $x(t)$ of Eq. (3) depends on states of $(-\infty, t]$. Obviously, the $\psi^{\gamma}$ Razumikhin-type stability theorem of Eq. (3) obtained in [17] can be applied to Eq. (1). However, the general asymptotic stability of Eq. (3), one of important stability criteria, has not been considered. In many cases, the general asymptotic stability of the equilibrium of systems is much more economically and practically feasible in contrast to the exponential stability and general decay $\psi^{\gamma}$ stability. Moreover, to the best of the authors' knowledge, so far no result has been concerned with Razumikhin-type theorems on the general asymptotic stability of Eq. (1).

In this paper, the Razumikhin-type $p$ th moment asymptotic stability of Eq. (1) is established without taking difficulties from infinite delay into account. On the other hand, as most NSFDEs could not be solved explicitly, numerical solutions have become essential. Efficient numerical methods for various NSFDEs can be found in [21-23]. In this paper, we develop the backward Euler method for Eq. (1). We show that the backward Euler method can preserve the asymptotic behavior of the mean square stability of exact solutions to Eq. (1).

The paper is organized as follows. In Section 2, we introduce some necessary notations and definitions. In Section 3, we establish the Razumikhin-type theorem on the pth moment asymptotic stability for Eq. (1). In Section 4, we study the mean square asymptotic stability of the backward Euler method for Eq. (1). Numerical experiments are presented in the finial section.

\section{Preliminaries}

Throughout this paper, unless otherwise specified, we use the following notations. Let $\left(\Omega, \mathcal{F},\left\{\mathcal{F}_{t}\right\}_{t \geq 0}, P\right)$ be a complete probability space with filtration $\left\{\mathcal{F}_{t}\right\}_{t \geq 0}$ satisfying the usual conditions (i.e., it is right continuous and $\mathcal{F}_{0}$ contains all $P$-null sets). $B(t)$ is an $r$-dimensional Brownian motion defined on the probability space. $|\cdot|$ denotes the Euclidean norm in $R^{n}$. The inner product of $x, y$ in $R^{n}$ is denoted by $\langle x, y\rangle$ or $x^{\top} y$. If $A$ is a vector or matrix, its transpose is denoted by $A^{\top}$. If $A$ is a matrix, its norm $\|A\|$ is defined by $\|A\|=\sup \{|A x|:|x|=1\}$. Let $t \geq 0$ and $C\left([q t, t] ; R^{n}\right)$ denote the family of all continuous functions $\xi$ from $[q t, t]$ to $R^{n}$ with the norm $\|\xi\|=\sup _{q t \leq \theta \leq t}|\xi(\theta)|$. For $p>0$, de- 
note by $L_{\mathcal{F}_{t}}^{p}\left([q t, t] ; R^{n}\right)$ the family of all $\mathcal{F}_{t}$-measurable, $C\left([q t, t] ; R^{n}\right)$-value random variables $\xi=\{\xi(\theta): q t \leq \theta \leq t\}$ with $E\|\xi\|^{p}<\infty$.

Consider an $n$-dimensional neutral stochastic pantograph equation

$$
d[x(t)-N(x(q t))]=f(t, x(t), x(q t)) d t+g(t, x(t), x(q t)) d B(t), \quad t \geq t_{0},
$$

with initial data $\left\{x(t): q t_{0} \leq t \leq t_{0}\right\}=\xi \in L_{\mathcal{F}_{0}}^{p}\left(\left[q t_{0}, t_{0}\right] ; R^{n}\right)$. Here $0<q<1, f:\left[t_{0}, \infty\right) \times$ $R^{n} \times R^{n} \rightarrow R^{n}, g:\left[t_{0}, \infty\right) \times R^{n} \times R^{n} \rightarrow R^{n \times r}$ and $N: R^{n} \rightarrow R^{n}$ are all continuous functions.

As usual, thought this paper, we assume that Eq. (4) has a unique global solution $x(t ; \xi)$ on $t \geq q t_{0}$ with $E\left(\sup _{q t_{0} \leq t<\infty}|x(t ; \xi)|^{p}\right)<\infty$. Moreover, we assume that $f(t, 0,0)=0$, $g(t, 0,0)=0, N(0)=0$ for the stability purpose, so that Eq. (4) admits a trivial solution $x(t ; 0)=0$.

The following assumption and definition will be used in the following sections.

Assumption (H) Assume that there is a constant $\kappa \in(0,1)$ such that

$$
|N(x)-N(y)| \leq \kappa|x-y|, \quad \forall x, y \in R^{n} .
$$

Definition 2.1 The trivial solution of Eq. (4) is said to be:

(1) $p$ th moment stable if, for every $\varepsilon>0$, there exists $\delta=\delta(\varepsilon)>0$ such that

$$
E|x(t ; \xi)|^{p} \leq \varepsilon, \quad \forall t \geq t_{0}
$$

whenever $E\|\xi\|^{p}<\delta$;

(2) $p$ th moment asymptotically stable if it is $p$ th moment stable and there exists a $\delta>0$ such that $E\|\xi\|^{p}<\delta$ implies

$$
\lim _{t \rightarrow \infty} E|x(t ; \xi)|^{p}=0
$$

(3) globally $p$ th moment asymptotically stable if it is $p$ th moment stable and, moreover, for all initial data $\xi \in L_{\mathcal{F}_{t_{0}}}^{p}\left(\left[q t_{0}, t_{0}\right] ; R^{n}\right)$,

$$
\lim _{t \rightarrow \infty} E|x(t ; \xi)|^{p}=0
$$

To study the stability of Eq. (4), we need to introduce some other notations. Let $C^{1,2}\left(\left[q t_{0}, \infty\right) \times R^{n} ; R_{+}\right)$denote the family of all nonnegative functions $V(t, x)$ on $\left[q t_{0}, \infty\right)$, which are once differentiable in $t$ and twice differentiable in $x$. Define an operator $L V$, associated with Eq. (4), acting on $V(t, x) \in C^{1,2}\left(\left[q t_{0}, \infty\right) \times R^{n} ; R_{+}\right)$by

$$
\begin{aligned}
L V(t, x, y)= & V_{t}(t, x-N(y))+V_{x}(t, x-N(y)) f(t, x, y) \\
& +\frac{1}{2} \operatorname{trace}\left[g^{\top}(t, x, y) V_{x x}(t, x-N(y)) g(t, x, y)\right]
\end{aligned}
$$

for $t \geq t_{0}$ and $x, y \in R^{n}$, where

$$
V_{t}=\frac{\partial V(t, x)}{\partial t}, \quad V_{x}=\left(\frac{\partial V(t, x)}{\partial x_{1}}, \frac{\partial V(t, x)}{\partial x_{2}}, \ldots, \frac{\partial V(t, x)}{\partial x_{n}}\right), \quad V_{x x}=\left(\frac{\partial^{2} V(t, x)}{\partial x_{i} \partial x_{j}}\right)_{n \times n}
$$


The following inequality (see Lemma 4.1 of chapter 6 in [24] ) will be used in this paper: Let $p \geq 1, a, b \in R^{n}$. Then for any $\varepsilon>0$,

$$
|a+b|^{p} \leq(1+\varepsilon)^{p-1}\left(|a|^{p}+\varepsilon^{1-p}|b|^{p}\right) .
$$

\section{Razumikhin-type theorem}

In this section, we investigate the $p$ th moment asymptotic stability of Eq. (4) by using the Razumikhin-type technique.

Theorem 3.1 Let $p \geq 1, c_{2} \geq c_{1}>0, \lambda>0$ and Assumption (H) hold. Assume that there exists a function $V(t, x) \in C^{1,2}\left(\left[q t_{0}, \infty\right) \times R^{n} ; R_{+}\right)$such that

$$
c_{1}|x|^{p} \leq V(t, x) \leq c_{2}|x|^{p}, \quad \forall(t, x) \in\left[q t_{0}, \infty\right) \times R^{n}
$$

and, moreover,

$$
E L V(t, \varphi(t), \varphi(q t)) \leq-\lambda E V(t, \varphi(t)-N(\varphi(q t))), \quad t \geq t_{0}
$$

for all $\varphi \in L_{\mathcal{F}_{t}}^{p}\left([q t, t] ; R^{n}\right)$ satisfying

$$
E V(q t, \varphi(q t))<\bar{p} E V(t, \varphi(t)-N(\varphi(q t))),
$$

where $\bar{p}>\frac{c_{2}}{c_{1}}(1-\kappa)^{-p}$. Then the trivial solution of Eq. (4) is globally pth moment asymptotically stable.

Proof For any given initial data $\xi \in L_{\mathcal{F}_{t_{0}}}^{p}\left(\left[q t_{0}, t_{0}\right] ; R^{n}\right)$, let $x(t)=x(t ; \xi)$ be the solution of the system (4).

(1) For any given $\varepsilon>0$, we will prove that there exists a $\delta>0$ such that $E\|\xi\|^{p}<\delta$ implies $E|x(t)|^{p} \leq \varepsilon$ for $t \geq t_{0}$. Let $\delta=\frac{c_{1}(1-\kappa)^{p}}{c_{2}(1+\kappa)^{p}} \varepsilon$. Using the inequality (5) with $\varepsilon=\kappa$ and Assumption $(\mathrm{H})$, we have

$$
\begin{aligned}
E\left|x\left(t_{0}\right)-N\left(x\left(q t_{0}\right)\right)\right|^{p} & \leq(1+\kappa)^{p-1}\left(E\left|x\left(t_{0}\right)\right|^{p}+\kappa^{1-p} E\left|N\left(x\left(q t_{0}\right)\right)\right|^{p}\right) \\
& \leq(1+\kappa)^{p-1}\left(E\left|x\left(t_{0}\right)\right|^{p}+\kappa E\left|x\left(q t_{0}\right)\right|^{p}\right) \\
& <(1+\kappa)^{p-1}(\delta+\kappa \delta) \\
& =(1+\kappa)^{p} \delta .
\end{aligned}
$$

Since $E|x(t)-N(x(q t))|^{p}$ is continuous, there exists a $T>t_{0}$ such that

$$
E|x(t)-N(x(q t))|^{p} \leq(1+\kappa)^{p} \delta \quad \text { for } t_{0} \leq t \leq T .
$$

Then, by (6),

$$
E V(t, x(t)-N(x(q t))) \leq c_{2}(1+\kappa)^{p} \delta \text { for } t_{0} \leq t \leq T .
$$

We assert that

$$
E V(t, x(t)-N(x(q t))) \leq c_{2}(1+\kappa)^{p} \delta \quad \text { for } t \geq t_{0} .
$$


We will prove (8) by contradiction. Suppose that there exists a $t^{*}>T$ such that

$$
E V\left(t^{*}, x\left(t^{*}\right)-N\left(x\left(q t^{*}\right)\right)\right)>c_{2}(1+\kappa)^{p} \delta
$$

Set $\bar{t}=\max \left\{t: E V(s, x(s)-N(x(q s))) \leq c_{2}(1+\kappa)^{p} \delta, s \in\left[t_{0}, t\right]\right\}$. Then there exists a sufficiently small $h>0$ such that

$$
\begin{aligned}
& q t \leq \bar{t} \quad \text { for } t \in(\bar{t}, \bar{t}+h] \\
& E V(t, x(t)-N(x(q t)))>c_{2}(1+\kappa)^{p} \delta \quad \text { for } t \in(\bar{t}, \bar{t}+h] .
\end{aligned}
$$

Using the inequality (5) with $\varepsilon=\frac{\kappa}{1-\kappa}$ and Assumption (H), we obtain that

$$
\begin{aligned}
E|x(t)|^{p} & \leq \frac{E|x(t)-N(x(q t))|^{p}}{(1-\kappa)^{p-1}}+\frac{E|N(x(q t))|^{p}}{\kappa^{p-1}} \\
& \leq \frac{E|x(t)-N(x(q t))|^{p}}{(1-\kappa)^{p-1}}+\kappa E|x(q t)|^{p} .
\end{aligned}
$$

Using (6) and (10), we have

$$
\begin{aligned}
E|x(t)|^{p} & \leq \frac{\sup _{t_{0} \leq s \leq \bar{t}} E|x(s)-N(x(q s))|^{p}}{(1-\kappa)^{p-1}}+\kappa \sup _{q t_{0} \leq s \leq \bar{t}} E|x(s)|^{p} \\
& \leq \frac{\sup _{t_{0} \leq s \leq \bar{t}} E V(s, x(s)-N(x(q s)))}{c_{1}(1-\kappa)^{p-1}}+\kappa \sup _{q t_{0} \leq s \leq \bar{t}} E|x(s)|^{p} \\
& \leq \frac{c_{2}(1+\kappa)^{p} \delta}{c_{1}(1-\kappa)^{p-1}}+\kappa \sup _{q t_{0} \leq s \leq \bar{t}} E|x(s)|^{p}
\end{aligned}
$$

for $t \in\left[t_{0}, \bar{t}\right]$, which also holds for $t \in\left[q t_{0}, t_{0}\right]$. Then we have

$$
\sup _{q t_{0} \leq t \leq \bar{t}} E|x(t)|^{p} \leq \frac{c_{2}(1+\kappa)^{p} \delta}{c_{1}(1-\kappa)^{p-1}}+\kappa \sup _{q t_{0} \leq t \leq \bar{t}} E|x(t)|^{p} .
$$

That is,

$$
\sup _{q t_{0} \leq t \leq \bar{t}} E|x(t)|^{p} \leq \frac{c_{2}(1+\kappa)^{p}}{c_{1}(1-\kappa)^{p}} \delta .
$$

Using (6) yields

$$
E V(t, x(t)) \leq \frac{c_{2}^{2}(1+\kappa)^{p} \delta}{c_{1}(1-\kappa)^{p}} \quad \text { for } t \in\left[q t_{0}, \bar{t}\right]
$$

Applying $\bar{p}>\frac{c_{2}}{c_{1}}(1-\kappa)^{-p},(9)$ and (12), we obtain that

$$
\begin{aligned}
\bar{p} E V(t, x(t)-N(x(q t))) & \geq \bar{p} c_{2}(1+\kappa)^{p} \delta>\frac{c_{2}^{2}(1+\kappa)^{p} \delta}{c_{1}(1-\kappa)^{p}} \\
& \geq E V(q t, x(q t)) \quad \text { for } t \in[\bar{t}, \bar{t}+h] .
\end{aligned}
$$


By Itô's formula and (7),

$$
E V(t, x(t)-N(x(q t))) \leq E V(\bar{t}, x(\bar{t})-N(x(q \bar{t}))) \leq c_{2}(1+\kappa)^{p} \delta \quad \text { for } t \in(\bar{t}, \bar{t}+h],
$$

which contradicts (9). Therefore (8) must hold. In a similar way to (11), we have

$$
\sup _{q t_{0} \leq s \leq t} E|x(s)|^{p} \leq \frac{c_{2}(1+\kappa)^{p} \delta}{c_{1}(1-\kappa)^{p}} \quad \text { for } t \geq t_{0} .
$$

That is,

$$
E|x(t)|^{p} \leq \frac{c_{2}(1+\kappa)^{p} \delta}{c_{1}(1-\kappa)^{p}}=\varepsilon \quad \text { for } t \geq t_{0} .
$$

The $p$ th moment stability is proved.

(2) By the same argument as shown in (1), we can obtain that for any initial data $\xi \in$ $L_{\mathcal{F}_{t_{0}}}^{p}\left(\left[q t_{0}, t_{0}\right] ; R^{n}\right)$, there exists a constant $H_{1}$ such that

$$
\begin{aligned}
& E V(t, x(t)-N(x(q t))) \leq H_{1} \quad \text { for } t \geq t_{0}, \\
& E|x(t)|^{p} \leq \frac{H_{1}}{c_{1}(1-\kappa)^{p}} \quad \text { for } t \geq t_{0}
\end{aligned}
$$

and

$$
E V(t, x(t)) \leq \frac{c_{2} H_{1}}{c_{1}(1-\kappa)^{p}} \quad \text { for } t \geq t_{0}
$$

We first show that

$$
\lim _{t \rightarrow \infty} E V(t, x(t)-N(x(q t)))=0 .
$$

In order to prove (13), it is enough to show that for any given $\gamma$ with $0<\gamma<H_{1}$, there exists a $T>0$ such that $E V(t, x(t)-N(x(q t)))<\gamma$ for $t>T$. Let $d>0$ satisfy $\bar{p} \frac{c_{1}}{c_{2}}(1-\kappa)^{p} \gamma>$ $\gamma+d /(1-\kappa)$. Obviously, $\bar{p} \frac{c_{1}}{c_{2}}(1-\kappa)^{p} s>s+d /(1-\kappa)$ for $s \geq \gamma$. Set $T_{0}=\frac{\bar{p} \frac{c_{1}}{c_{2}}(1-\kappa)^{p}-1}{\lambda(1-q)}, \bar{t}_{i}=\frac{t_{0}+T_{0}}{q^{2 i}}$, $\bar{s}_{i}=\frac{t_{0}+T_{0}}{q^{2 i+1}}, I_{i}=\left[\bar{s}_{i}, \bar{t}_{i+1}\right]$. Let $M$ be the smallest nonnegative integer such that $H_{1} \leq \gamma+M d$.

We will show that

$$
\begin{aligned}
& E V(t, x(t)-N(x(q t))) \leq \gamma+(M-i) d \quad \text { for } t \geq \bar{t}_{i}, i=0,1,2, \ldots, M, \\
& E|x(t)|^{p} \leq \frac{\gamma+(M-(i+1)) d+\left(1+\kappa+\kappa^{2}+\cdots+\kappa^{i}\right) d}{c_{1}(1-\kappa)^{p}} \\
& \quad \text { for } t \geq \bar{t}_{i}, i=0,1,2, \ldots, M, \\
& E V(t, x(t)) \leq c_{2} \frac{\gamma+(M-(i+1)) d+\left(1+\kappa+\kappa^{2}+\cdots+\kappa^{i}\right) d}{c_{1}(1-\kappa)^{p}} \\
& \quad \text { for } t \geq \bar{t}_{i}, i=0,1,2, \ldots, M .
\end{aligned}
$$


We will prove (14) by induction. Obviously, (14) holds for $i=0$. Assume that (14) holds for $1 \leq i \leq n<M$. Then we will obtain that there exists a $t^{*} \in I_{n}$ such that

$$
E V\left(t^{*}, x\left(t^{*}\right)-N\left(x\left(q t^{*}\right)\right)\right) \leq \gamma+(M-(n+1)) d
$$

Otherwise, for all $t \in I_{n}$, we have

$$
\begin{aligned}
& q t \geq \bar{t}_{n}, \\
& E V(t, x(t)-N(x(q t)))>\gamma+(M-(n+1)) d,
\end{aligned}
$$

which implies that

$$
\begin{aligned}
\bar{p} E V(t, x(t)-N(x(q t))) & \geq \bar{p}(\gamma+(M-(n+1)) d)>c_{2} \frac{\gamma+(M-(n+1)) d+\frac{d}{1-\kappa}}{c_{1}(1-\kappa)^{p}} \\
& >c_{2} \frac{\gamma+(M-(n+1)) d+\left(1+\kappa+\kappa^{2}+\cdots+\kappa^{n}\right) d}{c_{1}(1-\kappa)^{p}} \\
& \geq E V(q t, x(q t)) \quad \text { for } t \in I_{n} .
\end{aligned}
$$

Using Itô's formula, (7) and (16), we have

$$
\begin{aligned}
& E V\left(\bar{t}_{n+1}, x\left(\bar{t}_{n+1}\right)-N\left(x\left(q \bar{t}_{n+1}\right)\right)\right) \\
& \quad \leq E V\left(\bar{s}_{n}, x\left(\bar{s}_{n}\right)-N\left(x\left(q \bar{s}_{n}\right)\right)\right)-\lambda \int_{\bar{s}_{n}}^{\bar{t}_{n+1}} E V(t, x(t)-N(x(q t))) d t \\
& \quad \leq \gamma+(M-n) d-\lambda(\gamma+(M-(n+1)) d)\left(\bar{t}_{n+1}-\bar{s}_{n}\right) \\
& \quad \leq \gamma+(M-n) d-\left(\bar{p} \frac{c_{1}}{c_{2}}(1-\kappa)^{p}-1\right)(\gamma+(M-(n+1)) d) \\
& \quad \leq \gamma+(M-n) d-\frac{d}{1-\kappa}<\gamma+(M-(n+1)) d,
\end{aligned}
$$

which contradicts (16). So, (15) must hold. We assert that for all $t \geq t^{*}$,

$$
E V(t, x(t)-N(x(q t))) \leq \gamma+(M-(n+1)) d
$$

Otherwise, there is a $t>t^{*}$ such that

$$
E V(t, x(t)-N(x(q t)))>\gamma+(M-(n+1)) d .
$$

Set $\bar{t}=\max \left\{t: E V(s, x(s)-N(x(q s))) \leq \gamma+(M-(n+1)) d, s \in\left[t^{*}, t\right]\right\}$. Then there exists a sufficiently small $h>0$ such that

$$
\begin{aligned}
& q t \geq \bar{t}_{n} \quad \text { for } t \in[\bar{t}, \bar{t}+h] \\
& E V(t, x(t)-N(x(q t)))>\gamma+(M-(n+1)) d \quad \text { for } t \in(\bar{t}, \bar{t}+h],
\end{aligned}
$$


which implies that

$$
\begin{aligned}
\bar{p} E V(t, x(t)-N(x(q t))) & \geq \bar{p}(\gamma+(M-(n+1)) d)>c_{2} \frac{\gamma+(M-(n+1)) d+\frac{d}{1-\kappa}}{c_{1}(1-\kappa)^{p}} \\
& >c_{2} \frac{\gamma+(M-(n+1)) d+\left(1+\kappa+\kappa^{2}+\cdots+\kappa^{n}\right) d}{c_{1}(1-\kappa)^{p}} \\
& \geq E V(q t, x(q t)) \quad \text { for } t \in[\bar{t}, \bar{t}+h] .
\end{aligned}
$$

Then, by Itô's formula and (7),

$$
E V(\bar{t}+h, x(\bar{t}+h)-N(x(q(\bar{t}+h))))-E V(\bar{t}, x(\bar{t})-N(x(q \bar{t}))) \leq 0 .
$$

Hence $E V(\bar{t}+h, x(\bar{t}+h)-N(x(q(\bar{t}+h)))) \leq \gamma+(M-(n+1)) d$, which contradicts (21). We therefore have

$$
E V(t, x(t)-N(x(q t))) \leq \gamma+(M-(n+1)) d \quad \text { for } t \geq \bar{t}_{n+1} \geq t^{*} .
$$

On the other hand, using (6), (10) and (23), we can obtain that

$$
\begin{aligned}
E|x(t)|^{p} & \leq \frac{E|x(t)-N(x(q t))|^{p}}{(1-\kappa)^{p-1}}+\kappa E|x(q t)|^{p} \\
& \leq \frac{E V(t, x(t)-N(x(q t)))}{c_{1}(1-\kappa)^{p-1}}+\kappa E|x(q t)|^{p} \\
& \leq \frac{\gamma+(M-(n+1)) d}{c_{1}(1-\kappa)^{p-1}}+\kappa \frac{\gamma+(M-(n+1)) d+\left(1+\kappa+\kappa^{2}+\cdots+\kappa^{n}\right) d}{c_{1}(1-\kappa)^{p}} \\
& =\frac{\gamma+(M-(n+2)) d+\left(1+\kappa+\kappa^{2}+\cdots+\kappa^{n+1}\right) d}{c_{1}(1-\kappa)^{p}} \text { for } t \geq \bar{t}_{n+1} .
\end{aligned}
$$

Moreover, by (6),

$$
E V(t, x(t)) \leq c_{2} \frac{\gamma+(M-(n+2)) d+\left(1+\kappa+\kappa^{2}+\cdots+\kappa^{n+1}\right) d}{c_{1}(1-\kappa)^{p}}
$$

for $t \geq \bar{t}_{n+1}$. That is, (14) holds for $i=n+1$. By induction, (14) holds for $0 \leq i \leq M$. Thus

$$
E V(t, x(t)-N(x(q t))) \leq \gamma \quad \text { for } t \geq T=\bar{t}_{M} .
$$

So, (13) must hold. Using (6) yields

$$
\lim _{t \rightarrow \infty} E|x(t)-N(x(q t))|^{p}=0 .
$$

Since $E|x(t)|^{p} \leq \frac{H_{1}}{c_{1}(1-\kappa)^{p}}$, we conclude that $\limsup _{t \rightarrow \infty} E|x(t)|^{p}<\infty$. Using (10), we have

$$
\begin{aligned}
\limsup _{t \rightarrow \infty} E|x(t)|^{p} & \leq \frac{1}{(1-\kappa)^{p-1}} \lim _{t \rightarrow \infty} E|x(t)-N(x(q t))|^{p}+\kappa \limsup _{t \rightarrow \infty} E|x(q t)|^{p} \\
& =0+\kappa \limsup _{t \rightarrow \infty} E|x(t)|^{p},
\end{aligned}
$$


which implies that

$$
\limsup _{t \rightarrow \infty} E|x(t)|^{p}=0
$$

The proof is complete.

Remark The result obtained in Theorem 3.1 is different from the ones in $[2,16,17,19$, $20]$. The results obtained in $[2,16,19,20]$ only deal with NSFDEs with finite delay. It is easy to see that NSPE (4) is a class of NSFDEs with infinite delay. So, these results obtained in $[2,16,19,20]$ cannot be applied to NSPE (4). On the other hand, the result obtained in [17] studied the $\psi^{\gamma}$ stability of NSFDEs with infinite delay. But Theorem 3.1 studied the $p$ th moment asymptotic stability of NSPE (4). Since Theorem 3.1 is established without taking difficulties from infinite delay into account, the conditions of Theorem 3.1 are much weaker than the one obtained in [17]. So, our work is not trivial.

If we take $V(t, x)=|x|^{p}$, the condition (7) may be written as

$$
\begin{aligned}
E\left\{\frac { p } { 2 } | \varphi ( t ) - N ( \varphi ( q t ) ) | ^ { p - 4 } \left(| \varphi ( t ) - N ( \varphi ( q t ) ) | ^ { 2 } \left[2(\varphi(t)-N(\varphi(q t)))^{\top} f(t, \varphi(t), \varphi(q t))\right.\right.\right. \\
\left.\left.\left.\quad+|g(t, \varphi(t), \varphi(q t))|^{2}\right]+(p-2)\left|(\varphi(t)-N(\varphi(q t)))^{\top} g(t, \varphi(t), \varphi(q t))\right|^{2}\right)\right\} \\
\leq-\lambda E|\varphi(t)-N(\varphi(q t))|^{p}, \quad t \geq t_{0},
\end{aligned}
$$

for all $\varphi \in L_{\mathcal{F}_{t}}^{p}\left([q t, t] ; R^{n}\right)$ satisfying

$$
E|\varphi(q t)|^{p}<\bar{p} E|\varphi(t)-N(\varphi(q t))|^{p}
$$

where $\bar{p}>(1-\kappa)^{-p}$.

Corollary 3.1 Let Assumption $(\mathrm{H})$ hold. Assume that there are two positive constants $\lambda_{1}$ and $\lambda_{2}$ such that

$$
\begin{aligned}
E\left\{\frac { p } { 2 } | \varphi ( t ) - N ( \varphi ( q t ) ) | ^ { p - 4 } \left(| \varphi ( t ) - N ( \varphi ( q t ) ) | ^ { 2 } \left[2(\varphi(t)-N(\varphi(q t)))^{\top} f(t, \varphi(t), \varphi(q t))\right.\right.\right. \\
\left.\left.\left.\quad+|g(t, \varphi(t), \varphi(q t))|^{2}\right]+(p-2)\left|(\varphi(t)-N(\varphi(q t)))^{\top} g(t, \varphi(t), \varphi(q t))\right|^{2}\right)\right\} \\
\leq-\lambda_{1} E|\varphi(t)|^{p}+\lambda_{2} E|\varphi(q t)|^{p}
\end{aligned}
$$

for all $t \geq t_{0}$ and $\varphi \in L_{\mathcal{F}_{t}}^{p}\left([q t, t] ; R^{n}\right)$. If

$$
0<\kappa<\frac{1}{2} \quad \text { and } \quad \lambda_{1}>\frac{\lambda_{2}}{(1-2 \kappa)^{p}},
$$

then the trivial solution of Eq. (4) is globally pth moment asymptotically stable. 
Proof As in Theorem 3.1, let $x(t)=x(t ; \xi)$ be the solution of the system (4) for any given initial data $\xi \in L_{\mathcal{F}_{t_{0}}}^{p}\left(\left[q t_{0}, t_{0}\right] ; R^{n}\right)$. By the condition (27), we can choose $\bar{p}$ such that

$$
\frac{1}{\kappa^{p}}>\bar{p}>\frac{1}{(1-\kappa)^{p}} \quad \text { and } \quad \lambda_{1}>\frac{\lambda_{2} \bar{p}}{\left(1-\kappa(\bar{p})^{\frac{1}{p}}\right)^{p}}
$$

Let $t \geq 0$ and $x(t)$ satisfy

$$
E|x(q t)|^{p}<\bar{p} E|x(t)-N(x(q t))|^{p} .
$$

Note that for any $\varepsilon \in(0,1)$,

$$
\begin{aligned}
E|x(t)-N(x(q t))|^{p} & \leq \frac{1}{(1-\varepsilon)^{p-1}} E|x(t)|^{p}+\frac{1}{\varepsilon^{p-1}} E|N(x(q t))|^{p} \\
& \leq \frac{1}{(1-\varepsilon)^{p-1}} E|x(t)|^{p}+\frac{\kappa^{p} \bar{p}}{\varepsilon^{p-1}} E|x(t)-N(x(q t))|^{p} .
\end{aligned}
$$

Hence

$$
-E|x(t)|^{p} \leq-(1-\varepsilon)^{p-1}\left(1-\frac{\kappa^{p} \bar{p}}{\varepsilon^{p-1}}\right) E|x(t)-N(x(q t))|^{p} .
$$

It then follows from (26) and (29) that

$$
\begin{aligned}
E\left\{\frac { p } { 2 } | x ( t ) - N ( x ( q t ) ) | ^ { p - 4 } \left(| x ( t ) - N ( x ( q t ) ) | ^ { 2 } \left[2(x(t)-N(x(q t)))^{\top} f(t, x(t), x(q t))\right.\right.\right. \\
\left.\left.\left.\quad+|g(t, x(t), x(q t))|^{2}\right]+(p-2)\left|(x(t)-N(x(q t)))^{\top} g(t, x(t), x(q t))\right|^{2}\right)\right\} \\
\leq-\lambda_{1} E|x(t)|^{p}+\lambda_{2} E|x(q t)|^{p} \\
\leq-\left[\lambda_{1}(1-\varepsilon)^{p-1}\left(1-\frac{\kappa^{p} \bar{p}}{\varepsilon^{p-1}}\right)-\lambda_{2} \bar{p}\right] E|x(t)-N(x(q t))|^{2} \\
\quad=-\left[\lambda_{1}\left(1-\kappa(\bar{p})^{\frac{1}{p}}\right)^{p}-\lambda_{2} \bar{p}\right] E|x(t)-N(x(q t))|^{2}
\end{aligned}
$$

where $\varepsilon=\kappa(\bar{p})^{\frac{1}{p}}$. Using (28) yields

$$
\lambda_{1}\left(1-\kappa(\bar{p})^{\frac{1}{p}}\right)^{p}-\lambda_{2} \bar{p}>0 .
$$

That is, the condition (25) is satisfied. Hence the conclusion follows from Theorem 3.1. The proof is complete.

Corollary 3.2 Let Assumption $(\mathrm{H})$ hold. Assume that there are four positive constants $\lambda_{1}$, $\lambda_{2}, \lambda_{3}$ and $\lambda_{4}$ such that

$$
\begin{aligned}
& 2(x-N(y))^{\top} f(t, x, y) \leq-\lambda_{1}|x|^{2}+\lambda_{2}|y|^{2}, \\
& |g(t, x, y)|^{2} \leq \lambda_{3}|x|^{2}+\lambda_{4}|y|^{2}
\end{aligned}
$$


for all $t \geq t_{0}$ and $x, y \in R^{n}$. If

$$
0<\kappa<\frac{1}{2} \quad \text { and } \quad \lambda_{1}-\lambda_{3}>\frac{\lambda_{2}+\lambda_{4}}{(1-2 \kappa)^{2}}
$$

then the trivial solution of Eq. (4) is mean square stable.

Proof Using (31), we have

$$
\begin{aligned}
& E\left(2(\varphi(t)-N(\varphi(q t)))^{\top} f(t, \varphi(t), \varphi(q t))+|g(t, \varphi(t), \varphi(q t))|^{2}\right) \\
& \quad \leq-\left(\lambda_{1}-\lambda_{3}\right) E|\varphi(t)|^{2}+\left(\lambda_{2}+\lambda_{4}\right) E|\varphi(q t)|^{2}
\end{aligned}
$$

for all $\varphi \in L_{\mathcal{F}_{t}}^{p}\left([q t, t] ; R^{n}\right)$. Then the desired conclusion is obtained from Corollary 3.1 as taking $p=2$. The proof is complete.

\section{Mean square stability of the backward Euler method}

In this section, we develop the backward Euler method for Eq. (4). We will show that the backward Euler method can preserve the mean square stability of exact solutions of Eq. (4).

To define the backward Euler method for Eq. (4), we introduce a mesh $\bar{H}=\left\{m ; t_{-m}, \ldots\right.$, $\left.t_{-1}, t_{0}, t_{1}, \ldots, t_{m}, \ldots\right\}$ as follows. We assume that the initial time $t_{0}>0$. Set $t_{m}=q^{-1} t_{0}$. We define $m-1$ grid points $t_{1}<t_{2}<\cdots<t_{m-1}$ in $\left(t_{0}, t_{m}\right)$ by

$$
t_{i}=t_{0}+i \Delta_{0} \quad \text { for } i=1,2, \ldots, m-1
$$

where the initial step size $\Delta_{0}=\frac{t_{m}-t_{0}}{m}$, and define the other grid points by

$$
t_{k m+i}=q^{-k} t_{i} \quad \text { for } k=-1,0,1, \ldots, i=0,1,2, \ldots, m-1 .
$$

Let $h_{n}=t_{n+1}-t_{n}$. It is easy to see that grid points $t_{n}$ satisfy $q t_{n}=t_{n-m}$ for $n \geq 0$ and the step size $h_{n}$ satisfies

$$
q h_{n}=h_{n-m} \text { for } n \geq 0 \text { and } \lim _{n \rightarrow \infty} h_{n}=\infty
$$

For the given mesh $\bar{H}$, the backward Euler method for Eq. (4) is defined as follows:

$$
\begin{aligned}
& Y_{n+1}-N\left(Y_{n+1-m}\right) \\
& \quad=Y_{n}-N\left(Y_{n-m}\right)+h_{n} f\left(t_{n+1}, Y_{n+1}, Y_{n+1-m}\right)+g\left(t_{n}, Y_{n}, Y_{n-m}\right) \Delta B_{n}
\end{aligned}
$$

with the initial values

$$
Y_{n}=\xi\left(t_{n}\right), \quad n=-m,-m+1, \ldots, 0
$$

Here, $Y_{n}(n>0)$ is an approximation value of $x\left(t_{n}\right)$ and $\mathcal{F}_{t_{n}}$-measurable. $\Delta B_{n}=B\left(t_{n+1}\right)$ $B\left(t_{n}\right)$ is the Brownian motion increment. 
Definition 4.1 The numerical solution $Y_{n}(n=1,2, \ldots)$ of Eq. (4) is said to be mean square stable if for every $\varepsilon>0$, there exists $\delta=\delta(\varepsilon)>0$ such that

$$
E\left|Y_{n}\right|^{2} \leq \varepsilon, \quad n=1,2, \ldots,
$$

whenever the initial values satisfy $E\left(\max _{n=-m, \ldots, 0}\left|\xi\left(t_{n}\right)\right|^{2}\right)<\delta$ and, moreover,

$$
\lim _{n \rightarrow \infty} E\left|Y_{n}\right|^{2}=0
$$

Theorem 4.1 Assume the backward Euler method (33) is well defined.Let Assumption (H), conditions (31) and (32) hold. Then the backward Euler method approximate solution (33) is mean square stable.

Proof Set $\bar{Y}_{n}=Y_{n}-N\left(Y_{n-m}\right)$. For $n \geq 0$, by (33),

$$
\left|\bar{Y}_{n+1}-h_{n} f\left(t_{n+1}, Y_{n+1}, Y_{n+1-m}\right)\right|^{2}=\left|\bar{Y}_{n}+g\left(t_{n}, Y_{n}, Y_{n-m}\right) \Delta B_{n}\right|^{2} \text {. }
$$

Then we can obtain that

$$
\begin{aligned}
\left|\bar{Y}_{n+1}\right|^{2} \leq & \left|\bar{Y}_{n}\right|^{2}+2 h_{n}\left\langle\bar{Y}_{n+1}, f\left(t_{n+1}, Y_{n+1}, Y_{n+1-m}\right)\right\rangle+\left|g\left(t_{n}, Y_{n}, Y_{n-m}\right) \Delta B_{n}\right|^{2} \\
& +2\left\langle\bar{Y}_{n}, g\left(t_{n}, Y_{n}, Y_{n-m}\right) \Delta B_{n}\right\rangle
\end{aligned}
$$

which subsequently leads to

$$
\begin{aligned}
E\left|\bar{Y}_{n+1}\right|^{2} \leq & E\left|\bar{Y}_{n}\right|^{2}+2 h_{n} E\left(\bar{Y}_{n+1}, f\left(t_{n+1}, Y_{n+1}, Y_{n+1-m}\right)\right\rangle \\
& +E\left|g\left(t_{n}, Y_{n}, Y_{n-m}\right)\right|^{2} h_{n} .
\end{aligned}
$$

By the condition (31),

$$
\begin{aligned}
E\left|\bar{Y}_{n+1}\right|^{2} \leq & E\left|\bar{Y}_{n}\right|^{2}-\lambda_{1} h_{n} E\left|Y_{n+1}\right|^{2}+\lambda_{2} h_{n} E\left|Y_{n+1-m}\right|^{2} \\
& +\left(\lambda_{3} E\left|Y_{n}\right|^{2}+\lambda_{4} E\left|Y_{n-m}\right|^{2}\right) h_{n} .
\end{aligned}
$$

Using the equality $|a+b|^{2} \leq 2|a|^{2}+2|b|^{2}$ yields

$$
\left|\bar{Y}_{n+1}\right|^{2} \geq \frac{1}{2}\left|Y_{n+1}\right|^{2}-\left|N\left(Y_{n+1-m}\right)\right|^{2}
$$

and

$$
\left|\bar{Y}_{n}\right|^{2} \leq 2\left|Y_{n}\right|^{2}+2\left|N\left(Y_{n-m}\right)\right|^{2}
$$

Inserting these inequalities into (35) and using Assumption (H), we have

$$
\begin{aligned}
\left(\frac{1}{2}+\lambda_{1} h_{n}\right) E\left|Y_{n+1}\right|^{2} \leq & \left(2+\lambda_{3} h_{n}\right) E\left|Y_{n}\right|^{2}+\left(\kappa^{2}+\lambda_{2} h_{n}\right) E\left|Y_{n+1-m}\right|^{2} \\
& +\left(2 \kappa^{2}+\lambda_{4} h_{n}\right) E\left|Y_{n-m}\right|^{2}
\end{aligned}
$$


Let $A_{n}=1 / 2+\lambda_{1} h_{n}, B_{n}=2+\lambda_{3} h_{n}, C_{n}=\kappa^{2}+\lambda_{2} h_{n}$ and $D_{n}=2 \kappa^{2}+\lambda_{4} h_{n}$. Using these notations, (36) implies that

$$
E\left|Y_{n+1}\right|^{2} \leq \frac{B_{n}+C_{n}+D_{n}}{A_{n}} \max \left\{E\left|Y_{n}\right|^{2}, E\left|Y_{n+1-m}\right|^{2}, E\left|Y_{n-m}\right|^{2}\right\}
$$

By the condition (32), we have

$$
0<A=\lim _{n \rightarrow \infty} \frac{B_{n}+C_{n}+D_{n}}{A_{n}}=\frac{\lambda_{2}+\lambda_{3}+\lambda_{4}}{\lambda_{1}}<1 .
$$

Let $\varepsilon \in(0,1-A)$. There exists a positive integer $N$ such that

$$
\left|\frac{B_{n}+C_{n}+D_{n}}{A_{n}}-A\right|<\varepsilon, \quad n \geq N
$$

that is,

$$
\frac{B_{n}+C_{n}+D_{n}}{A_{n}}<A+\varepsilon<1, \quad n \geq N
$$

Set $C=\max \left\{\max _{0 \leq n \leq N}\left\{\frac{B_{n}+C_{n}+D_{n}}{A_{n}}\right\}, 1\right\}$. Then, by (37),

$$
\begin{aligned}
& E\left|Y_{n+1}\right|^{2} \leq C^{N+1} \max _{n=-m, \ldots, 0} E\left|Y_{n}\right|^{2}, \quad n \geq 0, \\
& E\left|Y_{n+1}\right|^{2} \leq(A+\varepsilon)^{\left[\frac{n-N}{m+1}\right]} \max _{-m \leq i \leq 0}\left\{E\left|Y_{N+i}\right|^{2}\right\}, \quad n \geq N,
\end{aligned}
$$

where [.] denotes the 'greatest integer in' function. (38) implies

$$
\lim _{n \rightarrow \infty} E\left|Y_{n}\right|^{2}=0
$$

The proof is complete.

\section{Numerical experiments}

In this section, we present numerical experiments to illustrate theoretical results obtained in the previous sections.

Consider the following scalar problem:

$$
\left\{\begin{array}{l}
d\left[x(t)-\frac{1}{4} \sin (x(0.5 t))\right]=(-12 x(t)+2 \sin (x(0.5 t))) d t+x(0.5 t) d B(t), \quad t \geq 1 \\
x(t)=1+2 \cos (t), \quad 0 \leq t \leq 1
\end{array}\right.
$$

For Eq. (39), we can obtain that $\kappa=1 / 4, \lambda_{1}=14, \lambda_{2}=3 / 2, \lambda_{3}=0$ and $\lambda_{4}=1$ corresponding to Corollary 3.2. By Corollary 3.2, the solution $x(t)$ of Eq. (39) is mean square stable. Theorem 4.1 shows that the backward Euler method approximation $Y_{n}$ of Eq. (39) can preserve the mean square stability of the exact solution $x(t)$.

A set of 20 blocks each containing 100 outcomes $\left(\omega_{i j}, 1 \leq i \leq 20,1 \leq j \leq 100\right)$ is simulated, and $Y_{n}\left(\omega_{i j}\right)$ denotes the $n$th step value of the backward Euler method approximation 

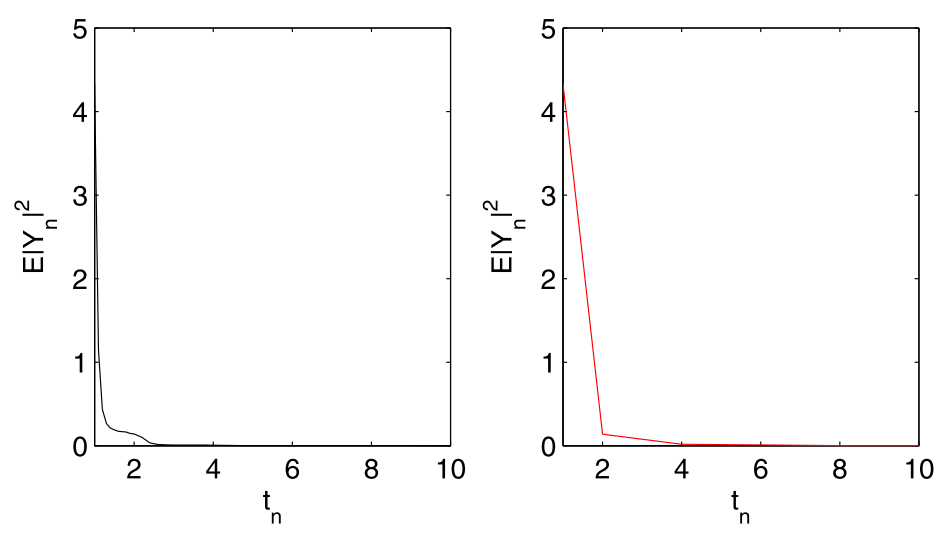

Figure 1 Asymptotic behavior of the backward Euler approximation with different initial step size $\Delta_{\mathbf{0}}$, left: $\boldsymbol{\Delta}_{\mathbf{0}}=\mathbf{0 . 1}$; right: $\boldsymbol{\Delta}_{\mathbf{0}}=\mathbf{1}$. The left one of Figure 1 describes the mean square asymptotic behavior of the backward Euler approximation $E\left|Y_{n}\right|^{2}$ of Eq. (39) with initial step size $\Delta_{0}=0.1$; the right one of Figure 1 describes the mean square asymptotic behavior of the backward Euler approximation $E\left|Y_{n}\right|^{2}$ of Eq. (39) with initial step size $\Delta_{0}=1$.

of the $j$ th trajectory in the $i$ th block. We compute $E\left|Y_{n}\right|^{2}$ of the backward Euler method approximation by

$$
E\left|Y_{n}\right|^{2} \cong \frac{1}{2,000} \sum_{i=1}^{20} \sum_{j=1}^{100}\left|Y_{n}\left(\omega_{i j}\right)\right| .
$$

Two tests are developed with different initial step sizes $\Delta_{0}=0.1,1$. Results are shown in Figure 1. It is easy to see that these results are compatible with the conclusion of Theorem 4.1.

\section{Conclusion}

In this paper, we have firstly investigated the $p$ th moment asymptotic stability of NSPEs. Although the NSPE is a special class of NFDEs with infinite delay, we show that the Razumikhin-type theorem on the $p$ th moment asymptotic stability of NSPEs can be established without taking into account difficulties from infinite delay. On the other hand, we also develop the backward Euler method for NSPEs. We show that the backward Euler method can preserve the mean square stability of exact solutions under suitable conditions. Numerical examples have been provided to demonstrate the validity of our results.

\section{Competing interests}

The author declares that he has no competing interests.

\section{Acknowledgements}

The author would like to thank the referees for their helpful comments and suggestions.

Received: 11 July 2012 Accepted: 7 June 2013 Published: 25 June 2013

\section{References}

1. Mao, X: Exponential stability in mean square of neutral stochastic differential functional equations. Syst. Control Lett. 26, 245-251 (1995)

2. Mao, X: Razumikhin-type theorems on exponential stability of neutral stochastic functional differential equations. SIAM J. Math. Anal. 28, 389-401 (1997)

3. Mao, X, Rodkina, A, Koroleva, N: Razumikhin-type theorems for neutral stochastic functional differential equations. Funct. Differ. Equ. 5(1-2), 195-211 (1998) 
4. Mao, X: Asymptotic properties of neutral stochastic differential delay equations. Stoch. Stoch. Rep. 68, 273-295 (2000)

5. Mao, X, Shen, Y, Yuan, C: Almost surely asymptotic stability of neutral stochastic differential delay equations with Markovian switching. Stoch. Process. Appl. 118, 1385-1406 (2008)

6. Fan, Z, Liu, M, Cao, W: Existence and uniqueness of the solutions and convergence of semi-implicit Euler methods for stochastic pantograph equations. J. Math. Anal. Appl. 325, 1142-1159 (2007)

7. Fan, Z, Song, M, Liu, M: The $\alpha$ th moment stability for the stochastic pantograph equation. J. Comput. Appl. Math 233, 109-120 (2009)

8. Iserles, A: On the generalized pantograph functional-differential equations. Eur. J. Appl. Math. 4, 1-38 (1993)

9. Huang, C, Vandewalle, S: Discretized stability and error growth of the nonautonomous pantograph equation. SIAM J. Numer. Anal. 42, 2020-2042 (2005)

10. Wang, W, Li, S: On the one-leg $h$-methods for solving nonlinear neutral functional differential equations. Appl. Math Comput. 193, 285-301 (2007)

11. Liu, Y: Asymptotic behaviour of functional-differential equations with proportional time delay. Eur. J. Appl. Math. 7, 11-30 (1996)

12. Koto, T: Stability of Runge-Kutta methods for the generalized pantograph equation. Numer. Math. 84, 233-247 (1999)

13. Bellen, $A$, Maset, $S$, Torelli, L: Contractive initializing methods for the pantograph equation of neutral type. In: Recent Trends in Numerical Analysis, vol. 3, pp. 35-41 (2000)

14. $\mathrm{Ma}, \mathrm{S}$, Yang, Z, Liu, M: $H_{\alpha}$-stability of modified Runge-Kutta methods for nonlinear neutral pantograph equations. J. Math. Anal. Appl. 335, 1128-1142 (2007)

15. Yu, Z: Almost surely asymptotic stability of exact and numerical solutions for neutral stochastic pantograph equations. Abstr. Appl. Anal. 2011, Article ID 143079 (2011). doi:10.1155/2011/143079

16. Zhou, S, Hu, S: Razumikhin-type theorems of neutral stochastic functional differential equations. Acta Math. Sci. 29(1), 181-190 (2009)

17. $\mathrm{Wu}, \mathrm{F}, \mathrm{Hu}, \mathrm{S}, \mathrm{Mao}, \mathrm{X}$ : Razumikhin-type theorem for neutral stochastic functional differential equations with unbounded delay. Acta Math. Sci. 31(4), 1245-1258 (2011)

18. Randjelovic, J, Jankovic, S: On the $p$-th moment exponential stability criteria of neutral stochastic functional differential equations. J. Math. Anal. Appl. 326, 266-280 (2007)

19. Jankovic, S, Randjelovic, J, Jovanovic, M: Razumikhin-type exponential stability criteria of neutral stochastic functional differential equations. J. Math. Anal. Appl. 355, 811-820 (2009)

20. Huang, L, Deng, F: Razumikhin-type theorems on stability of neutral stochastic functional differential equations. IEEE Trans. Autom. Control 53(7), 1718-1723 (2008)

21. Gan, S, Schurz, H, Zhang, H: Mean square convergence of stochastic $\theta$-methods for nonlinear neutral stochastic differential delay equations. Int. J. Numer. Anal. Model. 8, 201-213 (2011)

22. Zhou, S, Wu, F: Convergence of numerical solutions to neutral stochastic delay differential equations with Markovian switching. J. Comput. Appl. Math. 229, 85-96 (2009)

23. Yin, $\mathrm{B}, \mathrm{Ma}, \mathrm{Z}$ : Convergence of the semi-implicit Euler method for neutral stochastic delay differential equations with phase semi-Markovian switching. Appl. Math. Model. 35, 2094-2109 (2011)

24. Mao, X: Stochastic Differential Equations and Their Applications. Ellis Horwood, Chichester (1997)

doi:10.1186/1029-242X-2013-299

Cite this article as: Yu: Razumikhin-type theorem and mean square asymptotic behavior of the backward Euler method for neutral stochastic pantograph equations. Journal of Inequalities and Applications 2013 2013:299.

\section{Submit your manuscript to a SpringerOpen ${ }^{\circ}$ journal and benefit from:}

- Convenient online submission

Rigorous peer review

- Immediate publication on acceptance

- Open access: articles freely available online

- High visibility within the field

- Retaining the copyright to your article 